\title{
The Social and Cultural Context of Coping With Sickle Cell Disease: I. A Review of Biomedical and Psychosocial Issues
}

\author{
Oscar A. Barbarin \\ Marcelle Christian \\ University of Michigan
}

\begin{abstract}
Sickle cell disease (SCD) is widely conceived in the United States as a group of blood disorders that principally affect African Americans. Although pain is its principal feature, strokes, lung problems, sepsis, anxiety, depression, impaired social functioning, and maladjustment at work are frequent concomitants. This article selectively reviews biomedical and psychosocial aspects of SCD related to pain assessment, medical treatment, genetic counseling, education, and employment. The strongest support exists for claims of social deficits among adolescents and depression and work-related problems among adults. The social context of SCD, including issues related to socioeconomic status (SES), urbanicity, ethnicity, cultural values, and racial stigmatization, are important to include in empirical assessments and theoretical analyses of the effects of SCD on children and their families. The adverse psychosocial functioning often described as an effect of SCD might indeed be a consequence of these factors acting alone or in concert with the strains of SCD.
\end{abstract}

Since 1987, every child born in the state of Michigan is screened at birth for a variety of congenital genetically transmitted disorders. Among the diseases screened are several hemoglobinopathies grouped under the rubric of sickle cell disease (SCD) that have special significance for the African American community. SCD is a well-recognized source of distress in the lives of more than 50,000 African Americans and their families that often results in enduring psychosocial dysfunction (Evans, Burlew, \& Oler, 1988). Although it is unusual in the United States to find SCD among non-African Americans, the SCD trait does occur in a number of ethnic groups, whether

JOURNAL OF BLACK PSYCHOLOGY, Vol. 25 No. 3, August 1999 277-293

(C) 1999 The Association of Black Psychologists 
or not they identify as African American (Castro, Chicoye, Greenberg, Haynes, \& Peterson, 1994). Worldwide, these disorders are found among many different ethnic groups including those of Mediterranean, Caribbean, South and Central American, East Indian, and Arab descent. The aim of this article is to introduce themes emerging from psychosocial research on SCD and to argue the importance of broadening research paradigms on SCD to encompass social and cultural factors. This article begins with a description of SCD as biomedical and psychosocial phenomena. It reviews evidence on the physical and psychosocial consequences of SCD, the status of clinical practice with respect to its management, and limitations in current approaches to psychosocial research.

\section{BIOMEDICAL ASPECTS OF SCD}

\section{SCD AS A PHYSICAL DISORDER}

SCD is a family of blood disorders including sickle beta thalassemia and sickle cell anemia (SS and SC types). Persons with SCD have a preponderance of red blood cells that become hardened, brittle, and sickle-shaped when they release oxygen. This hardening and sickling of hemoglobin may result in vaso-occlusion, swelling, and pain episodes. These hemoglobins are fragile and do not live as long as normal red blood cells. Anemia is the consequence, then, not of cellular iron deficiency but rather of the abbreviated life span of the sickle hemoglobin. SCD can be accompanied by numerous physical symptoms and medical complications such as recurrent pain, anemia, low exercise tolerance, splenic sequestration, susceptibility to infections, lung problems, growth delay, late onset of puberty, strokes, priaprism, enuresis, and decreased life expectancy (Whitten \& Nishiura, 1985). Furthermore, patients can have acute and chronic pain syndromes. Physiological complications for children include pneumococcal infections, meningitis, osteomyelitis, cerebral vascular infarction, and enuresis (Stefanatou \& Bowler, 1997). The severity and the presenting symptoms associated with SCD vary greatly from individual to individual. Although professionals have failed to reach a consensus about definitions of severity in SCD, frequency of pain episodes, hospitalizations, infections, fevers, and swellings are primary markers used to gauge illness severity. 


\section{PAIN: A PRINCIPAL COMPONENT OF SCD}

Of all the possible indicators of severity in SCD, pain is accorded the greatest weight. This is true for many reasons but principally because it is the most common manifestation of and chief source of life disruption resulting from SCD. As a result of the disease, sickle cell patients can have pain in the lower back, legs, knees, chest, arms, and abdomen. Pain can be throbbing, sharp, or dull and can be unpredictable (Stefanatou \& Bowler, 1997). The painful vaso-occlusive crises of sickle cell anemia are caused by the sickling of the red blood cells that impedes their normal passage through the circulatory system (Stefanatou \& Bowler, 1997). Even though pain episodes are among the most common sequelae of SCD, obtaining reliable information needed to manage the pain still is difficult and elusive. Due to its subjective nature, methods for assessing pain are at best imprecise, and this imprecision is a foundation of interpersonal difficulties among persons with SCD, their families, and medical providers who must steer a course between over- and undermedication of pain. However, several different attempts have been made to develop a set of externally observable indicators of pain severity (Gil, Phillips, Edens, Martin, \& Abrams, 1993). Behaviors such as guarding, bracing, rubbing, grimacing, and sighing all can be observed and often indicate the level of pain. Walco and Dampier (1990) adopted a self-report approach called the Pediatric Pain Questionnaire (PPQ) to assess aspects of vaso-occlusive pain in children and adolescents with SCD. The PPQ includes body diagrams in which participants rate the specific sites and relative levels of pain intensity experienced. Patients also record whether they are "not hurting," "hurting a whole lot," or experiencing "severe pain." The PPQ also inquires about the degree to which pain interferes with activities. In addition, participants record the circumstances in which pain was likely to occur or increase. The measure proved to be a way of quantifying sickle cell pain, and observers were able to accurately perceive children's subjective pain ratings. Interrater agreement among physicians, parents, and children was high using this method for rating children's pain.

Although not diagnostic of pain, the use of children's drawings is another tool that provides a unique window to the emotional state of children in pain. The size of self-representational figures was found to be a useful indicator of pain (Stefanatou \& Bowler, 1997). Children drew smaller figures to represent themselves when they are in pain compared to when they are not in pain. This depiction suggests that children are so engrossed by pain that they feel "small" in the face of excruciating pain episodes. The different approaches 
represent creative efforts to assess severity of pain, but more research is needed to support the validity and utility of these methods.

\section{MEDICAL TREATMENT}

Because a cure for the disease itself has eluded researchers so far, medical care often centers on the use of analgesics to relieve pain, blood transfusions to minimize vaso-occlusion and strokes, and prophylactic penicillin in children under 5 years of age to prevent death from overwhelming infection. Shafer and Vichinsky(1994) review recent research evaluating two promising and widely used chemotherapies: opiods and hyroxyurea. Morphine infusion is widely used for children and adults with sickle cell vaso-occlusive crisis and is preferable to pain medications such as Demerol (which should be avoided because of its long-term ineffectiveness and multiple undesirable side effects). However, Shafer and Vichinsky caution that the continuous use of morphine and many other pain mediations for chronic pain has been associated with problems such as oversedation, acute chest syndrome, and hypoventilation and paradoxical effects. Hydroxyurea therapy has been shown to increase production of fetal hemoglobin. However, some concern has emerged about the cancer-causing effects of long-term use of hydroxyurea.

In addition to pharmacological treatments, there are discussions of surgical treatments for individuals with SCD. Blood transfusions have been proposed as a way of reversing the acute respiratory failure seen in sickle cell lung disease (Haynes, Manci, \& Voelkel, 1994). Chronic blood transfusions can decrease the frequency of acute chest syndrome in patients with lung disease, but because of the complications of multiple transfusions, the decision of when to recommend chronic transfusion remains an individual judgment (Haynes et al., 1994). In addition, for children with complications of SCD, bone marrow transplantation has been researched. In one study, children having a history of acute chest syndrome had a stabilization of lung functioning after transplantation (Walters et al., 1996). In this same study, patients with central nervous system vasculopathy also witnessed a stabilization of their cerebrovascular disease after transplantation. However, Shafer and Vichinsky (1994) comment that a variety of articles have been written concerning the controversy over the procedure and the controversy over the selection of candidates. Current criteria for consideration include history of acute chest syndrome, stroke, recurrent severe pain episodes, and early kidney or lung involvement. The potential risks of the transplantation procedure must be weighed against the possible benefits to the quality of life for individuals receiving the procedure (Shafer \& Vichinsky, 1994). 


\section{DISEASE SCREENING AND COUNSELING}

The symptoms and complications associated with SCD demonstrate convincingly its seriousness and its profound impact on quality of life. Given the high stakes involved, it is impossible to overstate the importance to individuals, couples, and families potentially affected by disease of early risk assessment through trait screening, prenatal diagnosis, and counseling after children have been diagnosed with the disease. However, Petrou, Brugiatelli, Ward, and Modell (1992) raise a number of ethical points that surround discussions of prenatal diagnosis. In this British study of couples at risk of having children with SCD, approximately $50 \%$ of the couples in which one partner actually had SCD requested prenatal diagnosis. More than $90 \%$ of referred couples who already had affected children requested prenatal diagnosis. As for the couples who declined prenatal diagnosis, many women in this study were deterred by the risk that screening had for their pregnancies. Fear of the procedure and religious objections also were cited as deterrents to screening (Petrou et al., 1992).

In addition to screening the fetus or the neonate, parents (or potential parents) are screened themselves. However, there are numerous impediments to having both parents genetically screened. In a sample of 60 families in which each family had a sickle cell trait newborn, for example, 60 mothers underwent counseling and testing (Coffee \& Vichinsky, 1993). By contrast, only 31 of the fathers $(51 \%)$ chose testing. The most common reasons given for the low participation among the fathers were that (a) the mother and father no longer were together, (b) the father works and could not get time off, (c) fear of needles and hospitals, (d) fear that testing might reveal more than hemoglobin trait (e.g., drug or alcohol abuse), and (e) incarceration of the father (Coffee \& Vichinsky, 1993). The issue of genetic counseling and screening mixes with a variety of impediments. Religious beliefs, logistics, and negative opinions of the medical profession in general all will have to be confronted as technological advances in genetic screening and counseling continue.

A legion of research scholars and administrators, including the four to whom this issue of the journal is dedicated, have made possible a number of exciting developments in the understanding and treatment of SCD. These include new procedures such as bone marrow transplants, unrelated cord blood transplants, new drugs, gene therapy, self-administration of intravenous pain medication, genetic screening, and psychological strategies for pain control. These developments hold out the promise of major breakthroughs in treatment and prevention of the many harmful sequelae of the disease. It is uncertain how many of these new developments will make their 
way into standard clinical practice. However, it is quite clear that the range of alternative treatment available to medical care staff and patients will be greatly expanded and will provide significant relief from, and even prevent adverse consequences of, the illness. In the meantime, much still needs to be done with respect to addressing the damaging psychosocial consequences of the disease. Many children and adults continue to suffer physically, socially, and emotionally. Until methods for cure and prevention become available, we are challenged to understand the psychosocial consequences of the disease and to build on insight from the behavioral and social sciences about ways in which to manage the disease more humanely, sensitively, and effectively to ensure that SCD compromises as little as possible the quality of life of children, adults, and families affected by the disease.

\section{PSYCHOSOCIAL ASPECTS OF SCD}

Knowledge about the long-term consequences of SCD for the psychological functioning of children, adults, and their families is expanding rapidly. There have been numerous articles on the psychological adjustment of children and adults with SCD. This includes social functioning, behavior problems, psychiatric diagnoses, and sexual functioning. Many of these studies have attempted to establish a relationship between ways of coping with illness, pain, and psychological adjustment and have suggested that patterns of coping by adults with SCD may be implicated in their experience of fear, guilt, loss of morale and zest for life, and a higher risk for marital dysfunction and chronic problems with employment. Recent tightening of eligibility requirements in federal safety net programs such as Supplemental Security Income has increased the importance of concern about academic and vocational adjustment and the controversy over claims attributing academic and intellectual deficits to SCD. A brief review of the existing evidence on adjustment outcomes provides a necessary background for a discussion of the social and cultural context of SCD.

\section{MENTAL HEALTH}

Thompson, Gil, Burbach, Keith, and Kinney (1993b) found that $64 \%$ of 7- to 12-year-old children with SCD were found to have parent-reported behavior problems. Half (50\%) of the children met the criteria for a psychiatric diagnosis, using a structured clinical interview based on the Diagnostic 
and Statistical Manual of Mental Disorders (American Psychiatric Association, 1994). Anxiety diagnoses and internal behavior problems were the most frequent types of adjustment problems (Thompson et al., 1993b). An unfortunate aspect of this study is that it does not adequately control for the effects of confounding variables such as SES, family structure, and economic hardship that might account for this extraordinarily high estimate of pathology. Similarly, Barbarin, Whitten, and Bonds (1994) report that symptoms of depression and anxiety are commonly reported for children and adolescents but that their rates are much closer to the one-third level most frequently reported for chronic illness. Importantly, neither research group included a comparison group in its study that would make it possible to determine whether the rates are higher among children with SCD than among children with similar SES and family backgrounds. It is possible that the observed psychopathology has more to do with social conditions of a select sample than with SCD per se.

In another study, a large proportion of children with SCD screened positive for the presence of mental disorders such as separation anxiety disorder, oppositional defiant disorder, attention deficit hyperactivity disorder, and enuresis (Cepeda, Yang, Price, \& Shah, 1997). In contrast to the Thompson et al. (1993b) data, only $31 \%$ of a group of sickle cell children screened positive for one or more of these disorders. This is much closer to the level of problems reported in epidemiological studies of the relationship between chronic illness and psychological disorders (Barbarin, 1990). However, the researchers in this study compared these rates to those of a control group consisting of same-race participants who were being seen in a nearby acute clinic for other acute problems such as ear infection and upper respiratory illness, and $42 \%$ of this control group screened positive for the presence of one or more mental disorders. This difference between mental disorders in the sickle cell and acute care groups was not significant (Cepeda et al., 1997). This study suggests that children and adolescents with SCD do not have an increased risk for clinically significant mental disorders than do same-race outpatient clinic controls. This research underscores the importance of using comparison groups in the investigation of problems in children with SCD.

Barrett et al. (1988) indicate that adult patients with SCD experience significant distress in the areas of employment and finances, sleeping and eating, and performance of normal daily activities. Anxiety and fear regarding body deterioration and a lack of assertiveness in social situations also were found. However, sickle cell patients in this study reported lower levels of sleep disturbance and disruption of normal activity than did other chronic pain populations. Problem severity in nearly all areas for persons with SCD 
was either lower or not significantly different from that for persons with other chronic illnesses. There were two important exceptions to this pattern: In finances and employment, patients with SCD experienced more severe problems. This finding highlights the importance of socioeconomic issues in programs intended to serve persons with SCD. Moreover, it reinforces the importance of appropriate comparison groups that control for socioeconomic situations in evaluating the deficits experienced by individuals with SCD.

Research on psychosocial adjustment of children with SCD has been wide-ranging. Psychosocial adjustment would include social relations, reports of anxiety, behavior problems, and other psychiatric diagnoses. For adults, evidence for high levels of anxiety and depression is convincing (Belgrave \& Molock, 1991). However, evidence for significant internalizing and externalizing disorders in children is at best inconsistent and inconclusive. The area of mental health impact that has the strongest support is the domain of social functioning (Barbarin, 1990; Utsey, 1991). Claims of severe emotional and behavioral adjustment problems due to SCD were not supported in a study by Noll, Vanatta, and Koontz (1996). Although those authors concluded that children with SCD are perceived as less sociable and less well accepted than their non-ill peers, no differences were observed regarding emotional well-being such as depression and self-concept. Girls with SCD were less well liked and were chosen less often by peers as best friends. However, males with SCD were perceived as being less aggressive than comparison peers. This suggests that youngsters with SCD are quite similar to peers and show a considerable amount of hardiness.

Similarly, although teachers reported more behavior problems among children with SCD than non-ill controls, the parents of children with SCD, children with sickle cell trait, and non-ill children did not differ in their ratings (Midence, McManus, Fuggle, \& Davies, 1996). Moreover, these children did not differ in self-reported depression or self-esteem (Midence et al., 1996). If consistent differences in social competence exist between children with SCD and non-ill children, then it is true primarily for older children or adolescents (Barbarin, 1999 [this issue]). Lemanek, Horwitz, and OheneFrempong (1994) report that 4- to 8-year-old children with SCD were found to be as socially competent as their peers in the comparison group of non-ill children. Similarly, Lemanek, Moore, Gresham, Williamson, and Kelley (1986) found no difference between Black and White children with sickle cell anemia and a comparison group of healthy controls who attended a family medical clinic for routine problems. In other research by Hurtig, Koepke, and Park (1989), the hypothesis that illness severity would affect psychosocial adjustment of children with SCD was not supported. In general, research 
seems to converge on the notion that although children with SCD do experience social and psychological problems, they are no more maladjusted or academically affected than other children growing up in similar impoverished environments.

\section{COPING AND PSYCHOLOGICAL ADJUSTMENT}

The failure to observe problems of adjustment in children with SCD does not imply the absence of illness-related stress. It is possible that many persons with SCD are coping effectively and reducing the level of problems observed in the group. How might various ways of coping with pain and illness affect the severity of psychosocial adjustment problems? A number of research articles have addressed this issue. In research by Gil, Williams, Thompson, and Kinney (1991), children and adolescents higher on negative thinking indexes were higher on overall levels of psychological distress as measured by the Child Assessment Scale, a semistructured diagnostic child interview. Adolescents high on negative thinking were more psychologically distressed on the Symptom Checklist-90-Revised than were adolescents low on negative thinking. In addition, children high on passive adherence had more frequent emergency room visits and significantly higher percentages of household, school, and social activity reduction (Gil et al., 1991). Furthermore, in other research, children and adolescents who become more negative in their thinking over time might come to rely more and more on health care services for their pain management (Gil, Thompson, et al., 1993). In Gil, Thompson, et al. (1993), children and adolescents high on coping attempts remained more active in a range of social, school, and household activities and had more uptime during painful episodes. Also, children and adolescents high on passive adherence had more frequent health care contacts.

Many of the same patterns are found among adults coping with sickle cell pain. Gil, Abrams, Phillips, and Keefe (1989) found that adults high on negative thinking and passive adherence had more severe pain, were less active, were more distressed, and used more health care services compared to adults without such patterns of thinking. A later study published by Gil, Abrams, Phillips, and Williams (1992) found that if levels of negative thinking and passive adherence increased from baseline to follow-up, then that increase was associated with even further reductions in the activity level during painful episodes. A number of additional studies replicating and expanding on these findings exist (Thompson, Gil, \& Abrams, 1996; Thompson, Gil, Abrams, \& Phillips, 1992, 1996). Research seems to converge on the idea that 
individuals can change adjustment classifications over time but that stable good adjustment is associated with lower levels of daily and illness-related stress, lower palliative methods of coping with stress, and less frequent pain coping strategies characterized by negative thinking (Thompson et al., 1992). In addition, a number of studies support the assertion that family characteristics can contribute to coping strategies. Poor maternal adjustment (Thompson, Gil, Burbach, Keith, \& Kinney, 1993a), a cohesive family environment (Kliewer \& Lewis, 1995), and family functioning characterized by high support and low conflict (Thompson et al., 1992) all can affect coping strategies used by individuals with SCD and their levels of reported psychological and physical adjustment.

\section{ACADEMIC PROBLEMS}

One of the most commonly cited consequences of SCD is impaired academic functioning, as reflected in poor teacher evaluations, below grade-level achievement, and grade retention (Barbarin et al., 1994). Hospitalization might be required to gain more rapid control over pain in a severe episode. As a consequence, children might experience disruptions in school attendance and be at risk for poor academic performance. They might be so distracted by pain that they cannot concentrate on schoolwork. However, the literature on academic performance has been inconsistent and controversial. For example, Brown, Armstrong, and Eckman (1993) suggest that subtle neurological deficits associated with cerebral vascular accidents affect cognitive processes and higher order cognitive abilities and that they later impede learning and academic achievement. However, in another example, Hurtig et al. (1989) found no evidence to support a relationship between illness severity and academic achievement. In addition, Midence et al. (1996) report no statistically significant difference in IQ between children with SCD and non-ill children. Richard and Burlew (1997) attempt to make sense of the inconsistencies in the SCD literature. First, they suggest that studies vary in the type of comparison group used. Comparison groups have included clinic clients, children with chronic illness, established norms, and siblings. In addition, they note that some researchers have taken measures to ensure that groups did not differ on important demographic variables such as race, age, and gender, whereas others have not. Clearly, it is difficult to assess whether the group differences on academic variables can be attributed to the children's medical condition or are confounded by differences in other demographic characteristics. 
In an effort to compare the academic performance of adolescents on reading and mathematics measures to that of an appropriate comparison group, Richard and Burlew (1997) enlisted 68 African American children attending a children's hospital in an urban midwestern city. The participants were 42 children with SCD in a sickle cell clinic and a comparison group of 26 healthy children who were attending a general medical clinic at the same hospital during the same period. Richard and Burlew comment that the two groups of children were similar on demographic characteristics such as gender, age, and socioeconomic indicators (e.g., parent education, marital status, occupation). They found no evidence of differences on grades in mathematics or reading, scores on standardized tests, or grade retention. Both the sickle cell and comparison groups had high rates of absenteeism, and the mean percentile scores in mathematics and reading were below the national average for their ages (Richard \& Burlew, 1997). Thus, it appears that children with SCD are no more at risk than are healthy children matched on demographic characteristics. Negative assumptions regarding the academic potential of children with SCD, and speculations that SCD produces neurological damage that results in poor academic performance, do not yet have a firm empirical foundation (Richard \& Burlew, 1997). The results of these studies underscore the importance of careful selection of comparison groups and speak to some of the controversy in the literature.

\section{EMPLOYMENT AND WORK ADJUSTMENT}

Academic and mental health problems are not the only manifestations of psychosocial dysfunction for adults with SCD. Career development and employment clearly are problematic for persons with SCD. Their difficulties unquestionably are exacerbated by the complications of SCD. Although there might be no real difference in the academic performance of African American children with SCD and that of African American children without SCD, persons with the illness experience disease-related physical limitations that constrain options for employment. For example, decreased capacity for exertion or strenuous activity might exist, and extreme weather, high altitudes, dangerous toxins, and infectious environments are more difficult to tolerate. Employability might be further restricted by a lack of preparation for skilled jobs, lack of access to employment in suburbs, and racial discrimination for persons with SCD just as it is for other non-Whites who do not have SCD. Thus, it is conceivable that SCD adds to the difficulty of finding and maintaining employment (Utsey, 1991). 


\section{IMPORTANCE OF THE SOCIAL AND CULTURAL CONTEXT OF ADJUSTMENT TO SCD}

\section{VIEWS OF SOCIAL CONTEXT OF SCD}

Mental health, educational attainment, and employment status are good barometers of adjustment to SCD. Unfortunately, in examining the factors that improve or impede adjustment, few researchers directly consider the potentially independent or compounding influences of ethnicity, racism, and economic inequality. These omissions significantly limit the capacity to design effective programs to manage the illness and enhance adjustment. The reasons for this oversight and the resulting gaps in the research literature on individual and family coping with SCD are puzzling. Accordingly, persons with SCD are defined and seen almost exclusively in terms of their medical condition. Research is funded by agencies that are more comfortable viewing illness in physical and medical terms. Social and psychological processes are not seen as determinative of important outcomes and, therefore, do not merit full consideration. As a consequence, the research that is designed omits consideration of how social factors shape the disease experience and determine patients' response to it.

CULTURE AND CLASS DIFFERENCES AS FEATURES OF THE SOCIAL CONTEXT OF SCD

The importance of the social context to disease management and adjustment is manifested in the tensions that often arise from ethnic, class, and power differences between care providers and patients (Pinderhughes, 1989). These insights have been discussed for some time and have implications beyond SCD. Extensive examination of these issues has taken place within broad professional discussion of ethnicity, racism, and cultural competence among care providers and their impact on the quality of service delivery in health and human services (Crawford, 1991; Ewalt, Freeman, Kirk, \& Poole, 1996). The visibility of these issues has risen as clinical care providers become more sensitized to the ways in which social class and ethnic differences potentially impede effective service delivery. Through a wealth of clinical examples, Pinderhughes (1989) adroitly demonstrates how unrecognized and unacknowledged racial, ethnic, and social class differences give rise to misperceptions and miscommunication between chronically ill persons and the health professionals who care for them. If not addressed, these 
misperceptions can become insurmountable obstacles that ultimately detract from the quality of care.

\section{EMERGENCY ROOMS AS ARENAS OF CULTURAL CLASH}

An example commonly cited by adults with SCD is the treatment they receive when they seek assistance for acute pain in hospital emergency rooms. Some staff lack experience and appropriate protocols for managing sickle cell pain episodes. The relationship and sense of collaboration often are strained by disagreements between patients and medical staff in the appraisal of pain. These disagreements devolve into conflict over how aggressively the pain should be treated. Suspicions about substance abuse make some care providers even more reluctant to treat pain vigorously. The problem is aggravated by the inherent subjectivity of pain and the dearth of "objective" information on which to base decisions about treatment. However, too often in arriving at decisions about responding to patient discomfort, medical personnel find it difficult to distinguish among addiction, dependence, tolerance, and pain. Furthermore, a lack of in-depth knowledge makes many staff reluctant to give patients the benefit of the doubt regarding pain relief. Interestingly, nurses might be less sympathetic to children hospitalized frequently for pain. Armstrong, Pegelow, Gonzalez, and Martinez (1992) found that nurses used lower doses of analgesics with children hospitalized more frequently for pain. The children in this study were non-ill actors coached in the expression of sickle cell pain, and after seeing these children discuss their pain on a videotape, nurses did not recommend an increase in medication, as the residents in the study tended to do. Armstrong et al. (1992) theorize that nurses, unlike residents, must respond to children's pain behavior on an ongoing basis with little opportunity to escape. The impact of repeated exposure to pain behavior might decrease nurses' responsiveness to children's pain behaviors. In addition, limited or inaccurate knowledge of nurses might contribute to faulty decision making (Armstrong et al., 1992).

\section{PITFALLS OF IGNORING SOCIAL CONTEXT OF SCD}

Ethnicity, class, and the social denigration associated with being in a lower status group clearly intersect with and influence medical staff decision making and, in turn, influence the nature of care provided. In addition, social factors such as urban poverty and its associated conditions amplify the stress experienced by persons with SCD and complicate management of the 
disorder. Omission of these issues from conceptions of adjustment, treatment, and consequences of SCD can be problematic, leading to faulty research conclusions. Questionable research claims include the attributions to SCD alone of outcomes such as academic failure, depression, and employment problems described earlier. Ultimately, such gaps in our knowledge undermine efforts to understand more fully the factors that influence the quality of care provided to patients. Stress, coping, outcomes, and management of SCD clearly are more likely to be understood if they are examined within the context of reciprocally reinforcing social factors such as cultural and value differences, inadequate resources, the denigration of African Americans, and the strains of urban living.

\section{CONCLUSION}

Challenges such as these to the validity of the psychosocial research on SCD have given rise to a systemic or bio-psychosocial perspective on the illness and its impact. Analyses emanating from this perspective emphasize the ways in which the biomedical aspects of the disease interact with family systems, culture, and socioeconomic factors to influence adjustment. Family members, cultural values, economic resources or hardship, and social identity possess the capacity to strengthen individual resolve to overcome the debilitating aspects of the illness and, alternatively, to generate guilt, shame, and anxiety that impede or undermine effective coping. Without question, more traditional, medically oriented research has made remarkable strides toward illuminating the physical and psychological aspects of SCD. Nevertheless, effective management of the disease will be hampered by misinformation, prejudices, uncertainty, and anxiety until we adopt a broader framework for understanding persons with SCD and their families. To deepen our understanding of adjustment to SCD, we must explore more fully the social context for coping with SCD and its cultural meanings within the African American community. This is an ambitious and intellectually challenging undertaking. We can claim only modest success in striving to address these questions. It is hoped that the articles in this special issue will prompt further research and encourage all of us to be critical of the literature we consume and conscious of how race and class both contribute to our understandingand often misunderstanding — of the issues facing individuals with SCD.

Challenges to dominant conceptions of SCD must themselves be placed in perspective. These questions arise within a context of considerable progress in understanding SCD and improvements in the services provided to 
individuals and families affected by the disorder. Together, researchers and clinicians working with sickle cell patients have devised methods for the screening and diagnosis of SCD, refined approaches for assessing pain, identified an array of medical and psychological complications, and designed surgical and pharmacological approaches to treat SCD. In the process, they have had to resolve a number of ethical and practical dilemmas related to genetic screening and the risks of addiction due to the treatment of chronic pain. Their work is far from complete. Even now, they are pushed to discover more objective ways in which to quantify pain, improve surgical and nonsurgical methods to enhance the quality of life for individuals with SCD, and discover ways in which to reduce the stigma often associated with genetic screening and counseling. Advances are being made as more and more psychosocial and neuropsychological research incorporates appropriate control groups to support claims about academic difficulties, behavior problems, and anxiety disorders in children and adolescents with SCD. It is hoped that this special issue and the research contributions it contains will advance our understanding of these important issues that have been underresearched and will serve as a stimulus to encourage additional work on the social context of coping with SCD.

\section{REFERENCES}

American Psychiatric Association. (1994). Diagnostic and statistical manual of mental disorders (4th ed.). Washington, DC: Author.

Armstrong, F. D., Pegelow, C. H., Gonzalez, J. C., \& Martinez, A. (1992). Impact of children's sickle cell history on nurse and physician ratings of pain and medication decisions. Journal of Pediatric Psychology, 17, 651-664.

Barbarin, O. (1990). Adjustment to serious childhood illness. In B. Lahey \& A. S. Kazdin (Eds.), Advances in Clinical Child Psychology (Vol. 13, pp. 377-403). New York: Plenum.

Barbarin, O. (1999). Do parental coping, involvement, religiosity, and racial identity mediate children's psychological adjustment to sickle cell disease? Journal of Black Psychology, 25, 391-426.

Barbarin, O., Whitten, C., \& Bonds, S. (1994). Estimating rates of psychosocial problems in urban and poor Children with sickle cell anemia. Health and Social Work, 19, 112-119.

Barrett, D., Wisotzek, I. E., Abel, G. G., Rouleau, J. L., Plat, A. F., Pollard, W. E., \& Eckman, J. R. (1988). Assessment of psychosocial functioning of patients with sickle cell disease. Southern Medical Journal, 81, 745-750.

Belgrave, F., \& Molock, S. (1991). The role of depression in hospital admissions and emergency treatment of patients with sickle cell disease. Journal of the National Medical Association, $83,777-781$

Brown, R. T., Armstrong, F. D., \& Eckman, J. R. (1993). Neurocognitive aspects of paediatric sickle cell disease. Journal of Learning Disabilities, 26, 33-45. 
Castro, O., Chicoye, L., Greenberg, J., Haynes, J., \& Peterson, K. (1994). Brighter horizons for sickle cell disease. Patient Care, 28, 26-44.

Cepeda, M. L., Yang, Y., Price, C., \& Shah, A. (1997). Mental disorders in children and adolescents with sickle cell disease. Southern Medical Journal, 90, 284-287.

Coffee, J., \& Vichinsky, E. (1993, May). The lack of paternal involvement in neonatal sickle cell screening follow-up. Paper presented at the meeting of the National Sickle Cell Disease Program, Philadelphia.

Crawford, M. (1991). The multicultural society: Sickle cell disease in children. Nursing, 4(31), 23-25.

Evans, R. C., Burlew, A. K., \& Oler, C. H. (1988). Children with sickle cell anemia: Parental relations, parent-child relations, and child behavior. Social Work, 33, 127-130.

Ewalt, P. L., Freeman, E. M., Kirk, S. A., \& Poole, D. L. (1996). Multicultural issues in social work. Washington, DC: NASW Press.

Gil, K. M., Abrams, M. R., Phillips, G., \& Keefe, F. J. (1989). Sickle cell disease pain: Relation of coping strategies to adjustment. Journal of Consulting and Clinical Psychology, 57, 725-731.

Gil, K. M., Abrams, M. R., Phillips, G., \& Williams, D. A. (1992). Sickle cell disease pain: II. Predicting health care use and activity level at 9-month follow up. Journal of Consulting and Clinical Psychology, 60, 267-273.

Gil, K. M., Phillips, G., Edens, J., Martin, N. J., \& Abrams, M. (1993). Observation of pain behaviors during episodes of sickle cell disease pain. Clinical Journal of Pain, 10, 128-132.

Gil, K. M ., Thompson, R. J., Keith, B. R., Totafaucette, M., Noll, S., \& Kinney, T. R. (1993). Sickle cell disease pain in children and adolescents: Change in pain frequency and coping strategies over time. Journal of Pediatric Psychology, 18, 621-637.

Gil, K. M ., Williams, D. A., Thompson, R. J., \& Kinney, T. R. (1991). Sickle cell disease in children and adolescents: The relation of child and parent pain coping strategies to adjustment. Journal of Pediatric Psychology, 16, 643-663.

Haynes, J., Manci, E., \& Voelkel, N. (1994). Pulmonary complications. In S. H. Embury, R. P. Hebbel, N. Mohandas, \& M. Steinberg (Eds.), Sickle cell disease: Basic principles and clinical practice (pp. 623-631). New York: Raven.

Hurtig, A. L., Koepke, D., \& Park, K. P. (1989). Relation between severity of chronic illness and adjustment in children and adolescents with sickle cell disease. Journal of Pediatric Psychology, 14, 117-132.

Kliewer, W., \& Lewis, H. (1995). Family influences on coping processes in children and adolescents with sickle cell disease. Journal of Pediatric Psychology, 20, 511-525.

Lemanek, K. L., Horwitz, W. L., \& Ohene-Frempong, K. (1994). A multi-perspective investigation of social competence in children with sickle cell disease. Journal of Pediatric Psychology, 19, 443-456.

Lemanek, K. L., Moore, S. L., Gresham, F. M., Williamson, D. A., \& Kelley, M. L. (1986). Psychological adjustment of children with sickle cell anemia. Journal of Pediatric Psychology, $11,397-410$

Midence, K., McManus, C., Fuggle, P., \& Davies, S. (1996). Psychological adjustment and family functioning in a group of British children with sickle cell disease: Preliminary empirical findings and a meta-analysis. British Journal of Clinical Psychology, 35, 439-450.

Noll, R. B., Vanatta, K., \& Koontz, K. (1996). Peer relationships and emotional well-being of youngsters with sickle cell disease. Child Development, 67, 423-436.

Petrou, M., Brugiatelli, M., Ward, R.H.T., \& Modell, B. (1992). Factors affecting the uptake of prenatal diagnosis for sickle cell disease. Journal of Medical Genetics, 29, 820-823. 
Pinderhughes, E. (1989). Understanding race, ethnicity, and power: The key to efficacy in clinical practice. New York: Free Press.

Richard, H. W., \& Burlew, A. K. (1997). Academic performance among children with sickle cell disease: Setting minimum standards for comparison groups. Psychological Reports, 81, 27-34.

Shafer, F. E., \& Vichinsky, E. (1994). New advances in the pathophysiology and management of sickle cell disease. Current Opinion in Hematology, 1, 125-135.

Stefanatou, A., \& Bowler, D. (1997). Depiction of pain in the self-drawings of children with sickle cell disease. Child: Care, Health, and Development, 23, 135-155.

Thompson, R. J., Gil, K. M., Abrams, M. R., \& Phillips, G. (1992). Stress, coping, and psychological adjustment of adults with sickle cell disease. Journal of Consulting and Clinical Psychology, 60, 433-440.

Thompson, R. J., Gil, K., Burbach, D. J., Keith, B. R., \& Kinney, T. R. (1993a). Psychological adjustment of mothers of children and adolescents with sickle cell disease: The role of stress, coping methods, and family functioning. Journal of Pediatric Psychology, 18, 549-559.

Thompson, R. J., Gil, K. M., Burbach, D. J., Keith, B. R., \& Kinney, T. R. (1993b). Role of child and maternal processes in the psychological adjustment of children with sickle cell disease. Journal of Consulting and Clinical Psychology, 61, 468-474.

Utsey, S. O. (1991). Vocational rehabilitation and counseling approaches with sickle cell anemia. Journal of Applied Rehabilitation Counseling, 22, 29-31.

Walco, G. A., \& Dampier, C. D. (1990). Pain in children and adolescents with sickle cell disease: A descriptive study. Journal of Pediatric Psychology, 15, 643-658.

Walters, M. C., Patience, M., Leisenring, W., Eckman, J., Scott, J. P., Mentzer, W. C., Davies, S. C., Ohene-Frempong, K., Bernaudin, F., Matthews, D. C., Storb, R., \& Sullivan, K. M. (1996). Bone marrow transplantation for sickle cell disease. New England Journal of Medicine, 335, 369-376.

Whitten, C., \& Nishiura, E. (1985). Sickle cell anemia: Public policy issues. In N. Hobbs \& J. Perrin (Eds.), Issues in the care of children with chronic illness (pp. 236-260). San Francisco: Jossey-Bass. 